\title{
Photochromic and Room Temperature Phosphorescent D-A Hybrid Crystals Regulated by Core-Substituted Naphthalenediimides
}

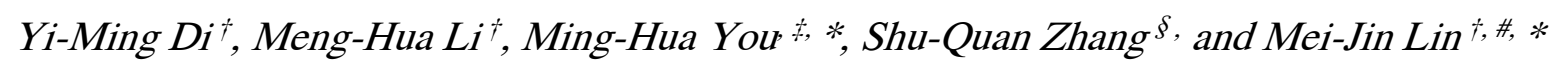

$\dagger$ Key Laboratory of Molecule Synthesis and Function Discovery (Fujian Province University), College of Chemistry, Fuzhou University, Fuzhou, 350108, China. E-mail: meijin_lin@fzu.edu.cn.

$\$$ College of Materials Science and Engineering, Fujian University of Technology, China, 350118. Email: 457443290@qq.com.

$\S$ College of Zhicheng, Fuzhou University, China, 350002.

\# College of Materials Science and Engineering, Fuzhou University, China, 350116

Table of Contents:

$\begin{array}{ll}\text { 1. Materials and instrumentations } & \text { S2 }\end{array}$

$\begin{array}{ll}\text { 2. X-ray crystallographic study } & \text { S2 }\end{array}$

$\begin{array}{ll}\text { 3. Molecular orbital calculation } & \text { S3 }\end{array}$

$\begin{array}{lll}\text { 4. } & \text { Related figures of hybrid } \mathbf{1} & \mathrm{S} 3\end{array}$

$\begin{array}{lll}\text { 5. PXRD patterns analysis } & \text { S4 }\end{array}$

$\begin{array}{lll}\text { 6. Infrared spectral analysis } & \text { S4 }\end{array}$

$\begin{array}{lll}\text { 7. Absorption spectra of organic ligands } & \text { S5 }\end{array}$

8. Crystal datum and structure refinement data $\quad$ S5

$\begin{array}{lr}9 . & \text { References } \\ \end{array}$ 


\section{Materials and instrumentations}

$\mathrm{H}_{3} \mathrm{PW}_{12} \mathrm{O}_{40}$, methanol $(\mathrm{MeOH})$ and $\mathrm{N}$-methylpyrrolidone (NMP) were obtained from commercial sources and were used without further purification. The organic ligand $\mathrm{H}_{2} \mathrm{BDMPy}-\mathrm{Br}_{2} \mathrm{NDI}$ and $\mathrm{H}_{2} \mathrm{BDMPy}-\mathrm{I}_{2} \mathrm{NDI}$ were synthesized following the literatures. ${ }^{1-3}$ The infrared spectra were obtained in the range of $400-4000 \mathrm{~cm}^{-1}$ from a PerkinElmer FT-IR spectrophotometer and power X-ray diffraction (PXRD) was recorded on a Rigaku MiniFlex-II X-Ray diffractometer. UV-vis diffuse reflectance spectra (DRS) were recorded on a Varian Cary 500 UV-vis spectrophotometer equipped with an integrating sphere at room temperature. The photochromism experiment was performed in air. The irradiation source was a $300 \mathrm{~W}$ xenon lamp equipped with a filter to provide a wavelength range of $420-780$ $\mathrm{nm}$. The distance between the light source and the sample was fixed at $15.0 \mathrm{~cm}$. Photoluminescence spectra and lifetimes at room temperature were recorded on an Edinburgh FLS 980 luminescence spectrometer. The photoluminescence quantum yields were measured on a Hamammatus C11347-11 absolute luminescence quantum yield measurement system equipped with an integrating sphere apparatus and a $150 \mathrm{~W}$ xenon light source. Electron paramagnetic resonance (EPR) measurements were obtained using a Bruker A300 instrument operating in the X-band at the room temperature.

\section{X-ray crystallographic study}

Crystal data for the complexes 1 and 2 were collected on a Rigaku Saturn 724 CCD diffractometer with Mo Ka radiation $(\lambda=0.71073 \AA)$ at $295 \mathrm{~K}$ and reduction were performed by using the program CrysAlisPro. ${ }^{4}$ The structures were solved by the direct method and different Fourier syntheses. All calculations were performed by full-matrix least-squares 
methods on $F^{2}$ by using the SHELXTL program, all non-hydrogen atoms were refined with anisotropic thermal parameters and the hydrogen atoms were fixed at calculated positions and refined by a riding mode..$^{5,6}$

\section{Molecular orbital calculation}

Molecular structure has been used for the evaluation of its molecular orbital energy through density functional theory (DFT) computations using the Gaussian 09 suite of programs. ${ }^{7} \mathrm{~A}$ hybrid functional, B3LYP, was adopted. Geometry was optimized using the LANL2DZ basis set. $^{8}$

\section{Related figures of hybrid 1}

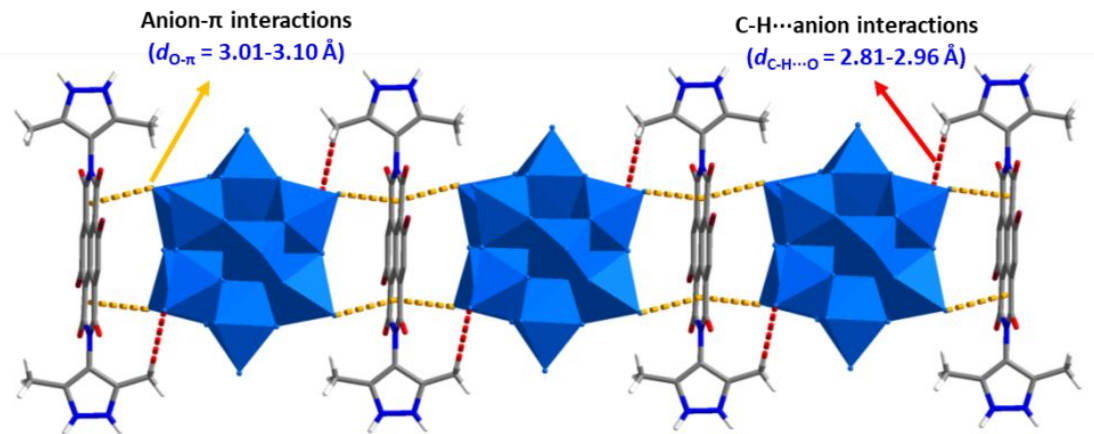

Figure S1. The 1-D long chain of hybrid 1.

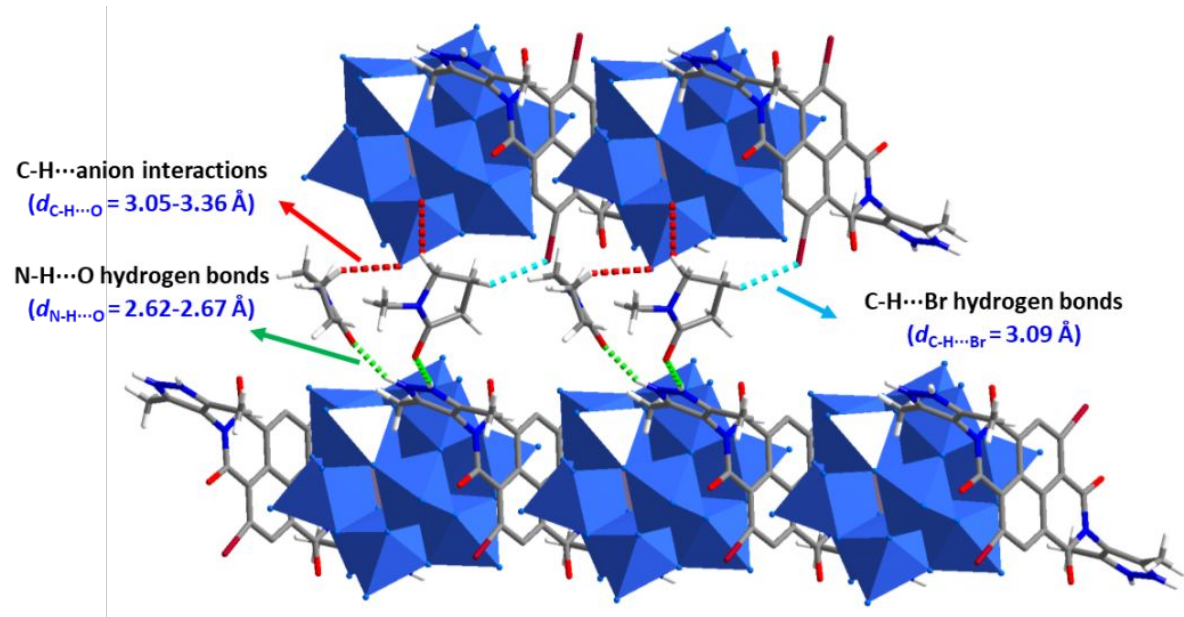

Figure S2. The hydrogen bonds between adjacent1-D long chains for hybrid $\mathbf{1}$. 


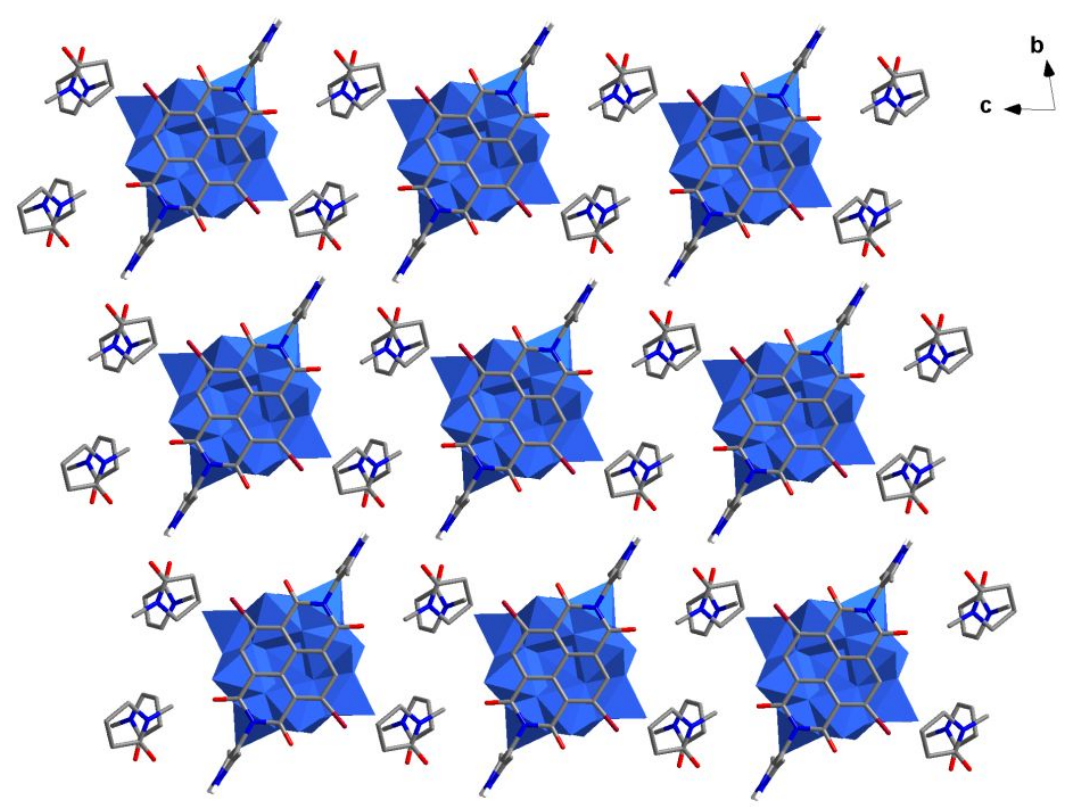

Figure S3. The supramolecular structure of hybrid $\mathbf{1}$ along a axis.

\section{PXRD patterns analysis}

(a)

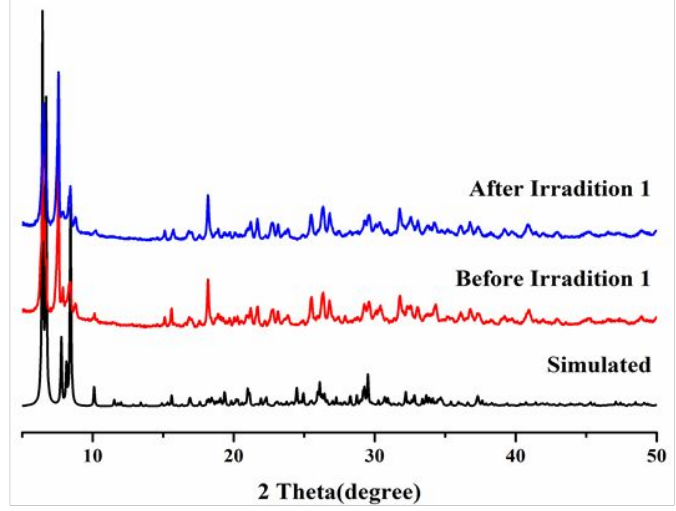

(b)

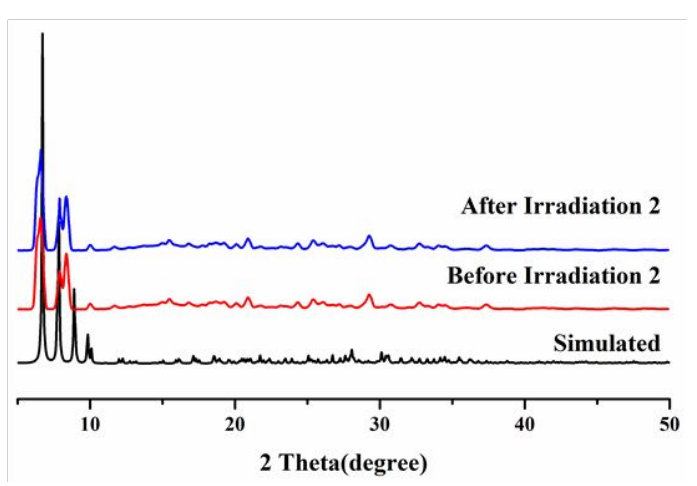

Figure S4. PXRD patterns of hybrids 1 (a) and 2 (b).

\section{Infrared spectral analysis}

(a)

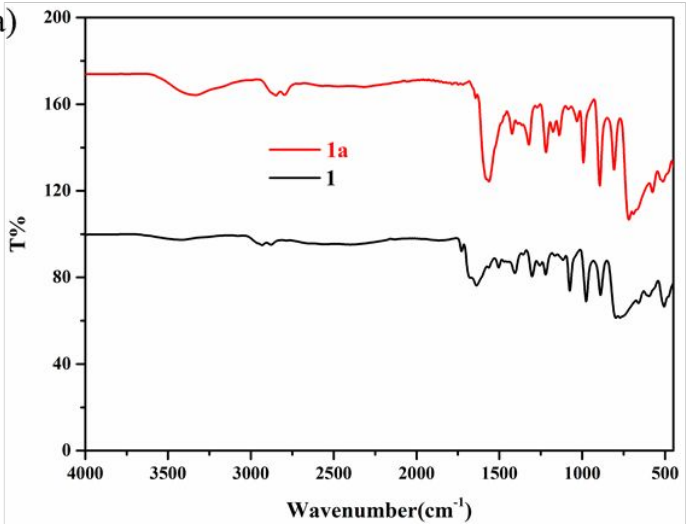

(b)

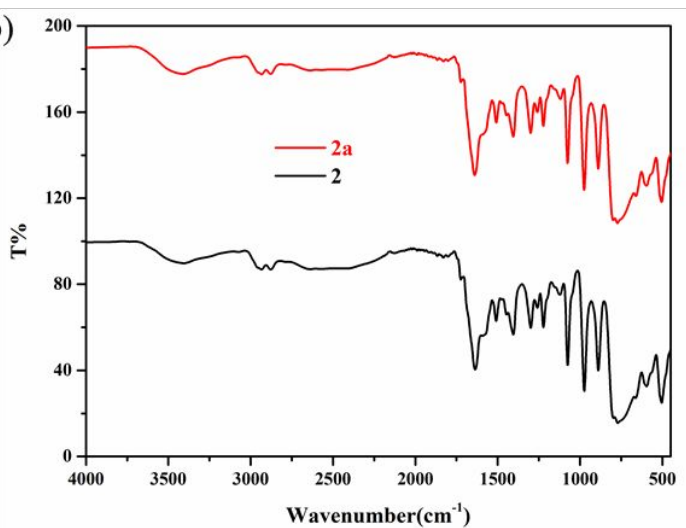

Figure S5. Infrared spectra of hybrids 1 (a) and 2 (b). 


\section{Absorption spectra of organic ligands}
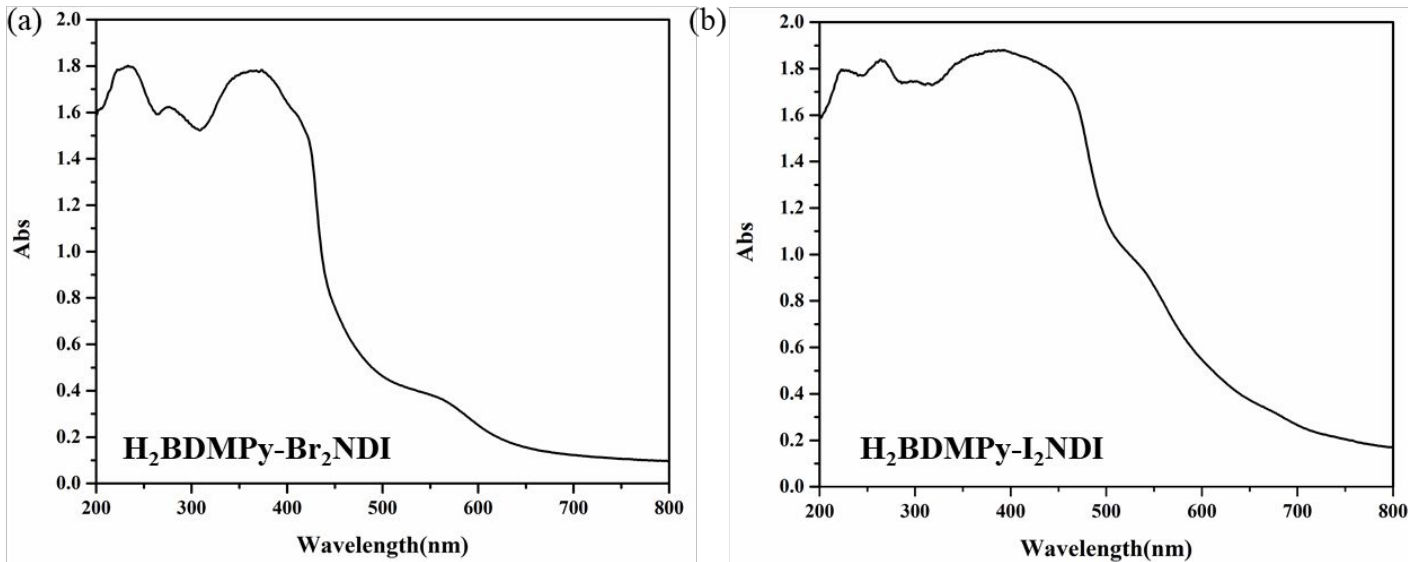

Figure S6. The absorption spectra of organic ligands $\mathrm{H}_{2} \mathrm{BDMPy}-\mathrm{Br}_{2} \mathrm{NDI}$ (a) and $\mathrm{H}_{2} \mathrm{BDMPy}-\mathrm{I}_{2} \mathrm{NDI}$ (b).

\section{Crystal datum and structure refinement data}

Table S1. Crystal datum and structure refinement parameters for the crystals $\mathbf{1}$ and $\mathbf{2}$

\begin{tabular}{|c|c|c|}
\hline Complexes & 1 & 2 \\
\hline Empirical formula & $\mathrm{C}_{44} \mathrm{H}_{52} \mathrm{Br}_{2} \mathrm{~N}_{10} \mathrm{O}_{48} \mathrm{PW}_{12}$ & $\mathrm{C}_{42} \mathrm{H}_{64} \mathrm{I}_{2} \mathrm{~N}_{9} \mathrm{O}_{48} \mathrm{PW}_{12}$ \\
\hline Formula weight & 3885.94 & 3585.41 \\
\hline Temperature/K & 296.15 & 296.15 \\
\hline Crystal system & triclinic & triclinic \\
\hline Space group & $P-1$ & $P-1$ \\
\hline $\mathrm{a} / \AA$ & 13.0871(18) & $13.2935(8)$ \\
\hline $\mathrm{b} / \AA ̊$ & $14.727(2)$ & $13.4723(8)$ \\
\hline $\mathrm{c} / \AA ̊$ & $15.153(2)$ & $15.1868(10)$ \\
\hline$\alpha /^{\circ}$ & $71.810(4)$ & $75.092(2)$ \\
\hline$\beta /{ }^{\circ}$ & $66.548(4)$ & $83.712(2)$ \\
\hline$\gamma /{ }^{\circ}$ & $65.680(4)$ & $70.447(2)$ \\
\hline Volume $/ \AA^{3}$ & $2402.8(6)$ & $2475.9(3)$ \\
\hline $\mathrm{Z}$ & 1 & 1 \\
\hline$\rho_{\text {calc }} \mathrm{g} / \mathrm{cm}^{3}$ & 2.686 & 2.405 \\
\hline$\mu / \mathrm{mm}^{-1}$ & 15.228 & 14.577 \\
\hline
\end{tabular}


$\mathrm{F}(000)$

Reflections collected

Independent reflections

Data/restraints/parameters

Goodness-of-fit on $\mathrm{F}^{2}$

$R_{1}^{a}, w R_{2}^{b}(I>2 \sigma(I))$

$R_{1}^{a}, w R_{2}^{b}$ (all data)
1743.0

1565.0

69179

43407

8414

8714

$8414 / 482 / 561$

$8714 / 18 / 423$

1.076

1.049

$\mathrm{R}_{1}=0.0956$,

$\mathrm{R}_{1}=0.0527$,

$\mathrm{wR}_{2}=0.2380$

$w_{2}=0.1288$

$\mathrm{R}_{1}=0.1208$

$\mathrm{R}_{1}=0.0682$

$\mathrm{wR}_{2}=0.2544$

$\mathrm{wR}_{2}=0.1399$

$$
R_{1}=\sum|| F_{0}|-| F_{\mathrm{c}}|| / \sum\left|F_{0}\right| \cdot w R_{2}=\left\{\sum\left[w\left(F_{0}^{2}-F_{\mathrm{c}}\right)^{2}\right] / \sum\left[w\left(F_{0}^{2}\right)^{2}\right]\right\}^{1 / 2}
$$

Table S2. Bond lengths for the crystal 1

\begin{tabular}{|c|c|c|c|c|c|c|c|}
\hline Atom & Length/Å & Atom & Length $/ \AA$ & Atom & Length/Å & Atom & Length/Å \\
\hline W001-O2 ${ }^{1}$ & $2.45(4)$ & $\mathrm{P} 008-\mathrm{O} 1^{1}$ & $1.54(4)$ & W004-O3 ${ }^{1}$ & $2.45(4)$ & C00M-C011 & $1.40(4)$ \\
\hline W001-O00A & $1.92(2)$ & P008-O1 & $1.54(4)$ & W004-O00K & $1.84(2)$ & C00Q-C00X & $1.40(4)$ \\
\hline W001-O4 & $2.46(4)$ & $\mathrm{P} 008-\mathrm{O} 3^{1}$ & $1.49(4)$ & W004-O00O & $1.90(2)$ & $\mathrm{C} 00 \mathrm{~V}-\mathrm{C} 00 \mathrm{Z}$ & $1.43(5)$ \\
\hline W001-O00B & $1.88(2)$ & P008-O3 & $1.49(4)$ & W004-O00S & $1.92(2)$ & $\mathrm{C} 00 \mathrm{~V}-\mathrm{C} 01 \mathrm{C}$ & $1.44(5)$ \\
\hline W001-O00C & $1.87(2)$ & $\mathrm{O} 2-\mathrm{O} 4^{1}$ & $1.59(5)$ & W004-O14 & $1.68(2)$ & $\mathrm{C} 00 \mathrm{X}-\mathrm{C} 013$ & $1.46(4)$ \\
\hline W001-O00D & $1.87(2)$ & $\mathrm{O} 1-\mathrm{O} 3^{1}$ & $1.82(5)$ & W005-O1 & $2.49(4)$ & C00Z-C018 & $1.30(5)$ \\
\hline W001-O00L & $1.66(2)$ & $\mathrm{Br} 07-\mathrm{Br} 00^{2}$ & $2.73(2)$ & W005-O00H & $1.67(2)$ & C018-C01B & $1.51(5)$ \\
\hline W002-O $2^{1}$ & $2.50(4)$ & Br07-C00Q & $1.89(3)$ & W005-O00N ${ }^{1}$ & $1.89(2)$ & $\mathrm{O} 016-\mathrm{C} 30$ & $1.30(7)$ \\
\hline W002-O00A & $1.86(2)$ & $\mathrm{Br} 00-\mathrm{C} 00 \mathrm{I}$ & $1.88(4)$ & W005-O00S & $1.86(2)$ & N20-C30 & $1.22(7)$ \\
\hline W002-O3 ${ }^{1}$ & $2.48(4)$ & O009-C00E & $1.22(4)$ & W005-O00 $\mathrm{Y}^{1}$ & $1.92(3)$ & N20-C34 & $1.42(8)$ \\
\hline W002-O00O & $1.91(2)$ & O00G-C013 & $1.24(4)$ & W005-O010 & $1.89(3)$ & $\mathrm{N} 20-\mathrm{C} 38$ & $1.46(6)$ \\
\hline W002-O00P & $1.67(2)$ & $\mathrm{N} 00 \mathrm{~F}-\mathrm{C} 00 \mathrm{E}$ & $1.37(4)$ & W006-O1 ${ }^{1}$ & $2.41(4)$ & $\mathrm{C} 01 \mathrm{D}-\mathrm{C} 01 \mathrm{H}$ & $1.58(8)$ \\
\hline W002-O00U & $1.89(2)$ & $\mathrm{N} 00 \mathrm{~F}-\mathrm{C} 00 \mathrm{Z}$ & $1.44(4)$ & W006-O00C & $1.90(2)$ & C01D-C34 & $1.54(7)$ \\
\hline W002-O010 & $1.89(3)$ & N00F-C013 & $1.40(4)$ & W006-O00J & $1.66(3)$ & $\mathrm{C} 01 \mathrm{H}-\mathrm{C} 30$ & $1.46(8)$ \\
\hline W003-O1 ${ }^{1}$ & $2.50(4)$ & N00W-N012 & $1.36(4)$ & W006-O00R & $1.88(2)$ & O01K-C019 & $1.17(7)$ \\
\hline W003-O00D ${ }^{1}$ & $1.90(2)$ & N00W-C018 & $1.35(4)$ & W006-O00U & $1.94(2)$ & N017-C019 & $1.33(8)$ \\
\hline W003-O00K & $1.95(2)$ & $\mathrm{N} 012-\mathrm{C} 00 \mathrm{~V}$ & $1.32(4)$ & W006-O00Y & $1.85(3)$ & N017-C01A & $1.40(7)$ \\
\hline W003-O00N & $1.89(3)$ & C00E-C011 & $1.51(4)$ & $\mathrm{P} 008-\mathrm{O} 2^{1}$ & $1.47(4)$ & N017-C35 & $1.41(7)$ \\
\hline W003-O00R & $1.85(2)$ & $\mathrm{C} 00 \mathrm{I}-\mathrm{C} 00 \mathrm{Q}^{2}$ & $1.39(5)$ & P008-O2 & $1.47(4)$ & C014-C35 & $1.46(2)$ \\
\hline W003-O13 & $1.67(2)$ & C00I-C011 & $1.35(5)$ & $\mathrm{P} 008-\mathrm{O} 4^{1}$ & $1.47(4)$ & C014-C39 & $1.39(2)$ \\
\hline W004-O4 ${ }^{1}$ & $2.52(4)$ & $\mathrm{C} 00 \mathrm{M}-\mathrm{C} 00 \mathrm{M}^{2}$ & $1.42(6)$ & P008-O4 & $1.47(4)$ & C019-C39 & $1.41(8)$ \\
\hline
\end{tabular}

12-X, 1-Y, 1-Z; ${ }^{2} 1-\mathrm{X}, 1-\mathrm{Y}, 1-\mathrm{Z}$ 
Table S3. Bond lengths for the crystal 2

\begin{tabular}{|c|c|c|c|c|c|c|c|}
\hline Atom & Length/Å & Atom & Length/Å & Atom & Length/Å & Atom & Length/Å \\
\hline W001-O00D & $1.892(11)$ & $\mathrm{W} 006-\mathrm{O} 2^{1}$ & $2.399(19)$ & W006-O2 ${ }^{1}$ & $1.918(13)$ & $\mathrm{C} 00 \mathrm{~J}-\mathrm{C} 00 \mathrm{R}^{2}$ & $1.37(2)$ \\
\hline W001-O00E & $1.875(15)$ & W006-O014 & $1.890(15)$ & W006-O014 & $2.500(16)$ & C00K-C00Q & $1.388(18)$ \\
\hline W001-O00F & $1.874(13)$ & W006-O016 & $1.869(15)$ & W006-O016 & $2.427(18)$ & $\mathrm{C} 00 \mathrm{~K}-\mathrm{C} 00 \mathrm{~T}$ & $1.45(2)$ \\
\hline W001-O00L & $1.880(11)$ & I07-C00R & $2.126(12)$ & I07-C00R & $1.890(15)$ & C00P-C00Q & $1.423(17)$ \\
\hline W001-O00X & $1.673(10)$ & $\mathrm{P} 008-\mathrm{O} 2^{1}$ & $1.60(2)$ & $\mathrm{P} 008-\mathrm{O} 2^{1}$ & $1.674(11)$ & C00P-C00R & $1.378(19)$ \\
\hline W001-O4 ${ }^{1}$ & $2.465(15)$ & $\mathrm{P} 008-\mathrm{O} 2$ & $1.60(2)$ & P008-O2 & $1.902(13)$ & $\mathrm{C} 00 \mathrm{P}-\mathrm{C} 00 \mathrm{~V}$ & $1.47(2)$ \\
\hline $\mathrm{W}_{001-O 1^{1}}$ & $2.397(18)$ & $\mathrm{P} 008-\mathrm{O} 4^{1}$ & $1.484(16)$ & $\mathrm{P} 008-\mathrm{O} 4^{1}$ & $2.429(17)$ & $\mathrm{C} 00 \mathrm{Q}-\mathrm{C} 00 \mathrm{Q}^{2}$ & $1.45(3)$ \\
\hline W002-O00D & $1.875(11)$ & P008-O4 & $1.484(16)$ & P008-O4 & $1.874(13)$ & $\mathrm{O} 2-\mathrm{O} 4{ }^{1}$ & $1.80(2)$ \\
\hline W002-O00I ${ }^{1}$ & $1.857(15)$ & $\mathrm{P} 008-\mathrm{O} 1^{1}$ & $1.498(17)$ & $\mathrm{P} 008-\mathrm{O} 1^{1}$ & $1.902(14)$ & N010-N011 & $1.34(2)$ \\
\hline W002-O00M & $1.913(14)$ & P008-O1 & $1.498(17)$ & P008-O1 & $1.662(11)$ & N010-C017 & $1.43(3)$ \\
\hline W002-O00S & $1.906(12)$ & P008-O3 & $1.533(18)$ & P008-O3 & $1.854(14)$ & N011-C018 & $1.38(2)$ \\
\hline W002-O41 & $2.471(15)$ & $\mathrm{P} 008-\mathrm{O} 3^{1}$ & $1.533(18)$ & $\mathrm{P} 008-\mathrm{O} 3^{1}$ & $1.856(13)$ & C015-C017 & $1.39(2)$ \\
\hline W002-O15 & $1.686(10)$ & $\mathrm{O} 00 \mathrm{~A}-\mathrm{C} 00 \mathrm{~V}$ & $1.226(16)$ & $\mathrm{O} 00 \mathrm{~A}-\mathrm{C} 00 \mathrm{~V}$ & $2.482(17)$ & C015-C018 & $1.41(3)$ \\
\hline $\mathrm{W}_{002-\mathrm{O}}{ }^{1}$ & $2.416(17)$ & O00B-C00T & $1.222(16)$ & O00B-C00T & $1.905(13)$ & $\mathrm{C} 017-\mathrm{C} 20$ & $1.45(3)$ \\
\hline W003-O00L & $1.910(13)$ & N00G-C00T & $1.391(17)$ & N00G-C00T & $1.888(14)$ & C018-C0AA & $1.49(3)$ \\
\hline W003-O00N & $1.681(10)$ & N00G-C00V & $1.439(19)$ & N00G-C00V & $1.874(14)$ & O4-O1 & $1.60(2)$ \\
\hline W003-O00O & $1.878(14)$ & N00G-C015 & $1.42(2)$ & N00G-C015 & $1.910(14)$ & O4-O3 & $1.66(2)$ \\
\hline W003-O00S ${ }^{1}$ & $1.879(13)$ & $\mathrm{C} 00 \mathrm{~J}-\mathrm{C} 00 \mathrm{~K}$ & $1.371(18)$ & $\mathrm{C} 00 \mathrm{~J}-\mathrm{C} 00 \mathrm{~K}$ & $1.647(14)$ & $\mathrm{O} 1-\mathrm{O} 3^{1}$ & $1.72(2)$ \\
\hline
\end{tabular}

${ }^{1} 1-\mathrm{X}, 1-\mathrm{Y}, 1-\mathrm{Z} ;{ }^{2} 2-\mathrm{X}, 1-\mathrm{Y}, 1-\mathrm{Z}$

Table S4. Bond angles for the crystal 1

\begin{tabular}{|c|c|c|c|c|c|c|c|}
\hline Atom & Angle ${ }^{\circ}$ & Atom & Angle $/^{\circ}$ & Atom & Angle $/^{\circ}$ & Atom & Angle $/^{\circ}$ \\
\hline $\mathrm{O} 2^{1}-\mathrm{W} 001-\mathrm{O} 4$ & $37.8(13)$ & $\mathrm{O} 2^{1}-\mathrm{P} 008-\mathrm{O} 3^{1}$ & $70(2)$ & $\mathrm{O}^{00 \mathrm{~B}^{1-} \mathrm{W} 004-\mathrm{O} 3^{1}}$ & $93.7(12)$ & W002-O00U-W006 & $137.3(14)$ \\
\hline O00A-W001-O2 ${ }^{1}$ & $63.4(11)$ & $\mathrm{O} 2-\mathrm{P} 008-\mathrm{O} 3^{1}$ & $110(2)$ & $\mathrm{O}^{0} \mathrm{~B}^{1}-\mathrm{W} 004-\mathrm{O} 00 \mathrm{~S}$ & $88.1(11)$ & W006-O00Y-W005 & $141.0(14)$ \\
\hline O00A-W001-O4 & $90.7(11)$ & O4-P008-O2 & $115(2)$ & $\mathrm{O} 3^{1}-\mathrm{W} 004-\mathrm{O} 4^{1}$ & $39.3(13)$ & W005-O010-W002 & $139.7(13)$ \\
\hline O00B-W001-O2 ${ }^{1}$ & $93.4(12)$ & $\mathrm{O} 4^{1}-\mathrm{P} 008-\mathrm{O} 2^{1}$ & $115(2)$ & O00K-W004-O4 ${ }^{1}$ & $65.1(11)$ & C00Q-Br07-Br002 & $67.8(11)$ \\
\hline O00B-W001-O00A & $155.9(9)$ & O41-P008-O2 & $65(2)$ & O00K-W004-O00B ${ }^{1}$ & $87.6(11)$ & C00E-NOOF-C00Z & $116(3)$ \\
\hline O00B-W001-O4 & $65.6(11)$ & $\mathrm{O} 4-\mathrm{P} 008-\mathrm{O} 2^{1}$ & $65(2)$ & O00K-W004-O3 ${ }^{1}$ & $90.1(11)$ & C00E-N00F-C013 & $125(3)$ \\
\hline O00C-W001-O2 ${ }^{1}$ & $64.9(11)$ & O4-P008-O4 ${ }^{1}$ & $180(3)$ & O00K-W004-O00O & $85.8(10)$ & C013-N00F-C00Z & $118(3)$ \\
\hline O00C-W001-O00A & $86.9(10)$ & $\mathrm{O} 4^{1}-\mathrm{P} 008-\mathrm{O} 1^{1}$ & $74(2)$ & O00K-W004-O00S & $154.0(10)$ & C018-N00W-N012 & $109(3)$ \\
\hline O00C-W001-O4 & $90.5(12)$ & O41-P008-O1 & $106(2)$ & O00O-W004-O4 & $93.3(11)$ & C00V-N012-N00W & $109(3)$ \\
\hline O00C-W001-O00B & $88.9(11)$ & O4-P008-O1 & $74(2)$ & O00O-W004-O00B ${ }^{1}$ & $156.9(10)$ & O009-C00E-N00F & $122(3)$ \\
\hline O00D-W001-O $2^{1}$ & $90.3(11)$ & O4-P008-O1 ${ }^{1}$ & $106(2)$ & O00O-W004-O3 ${ }^{1}$ & $64.3(12)$ & O009-C00E-C011 & $120(3)$ \\
\hline O00D-W001-O00A & $86.4(10)$ & $\mathrm{O}^{1}{ }^{1}-\mathrm{P} 008-\mathrm{O} 3^{1}$ & $69(2)$ & O00O-W004-O00S & $88.3(10)$ & N00F-C00E-C011 & $118(3)$ \\
\hline O00D-W001-O4 & $65.2(11)$ & O41-P008-O3 & $111(2)$ & O00S-W004-O41 & $90.2(12)$ & $\mathrm{C} 00 \mathrm{Q} 2-\mathrm{C} 00 \mathrm{I}-\mathrm{Br} 00$ & $108(2)$ \\
\hline
\end{tabular}




\begin{tabular}{|c|c|c|c|c|c|c|c|}
\hline O00D-W001-O00B & $87.3(10)$ & O4-P008-O3 ${ }^{1}$ & $111(2)$ & O00S-W004-O3 ${ }^{1}$ & $64.7(11)$ & C011-C00I-Br00 & $129(3)$ \\
\hline O00D-W001-O00C & $154.7(10)$ & O4-P008-O3 & $69(2)$ & O14-W004-O4 ${ }^{1}$ & $160.2(14)$ & $\mathrm{C} 011-\mathrm{C} 00 \mathrm{I}-\mathrm{C} 00 \mathrm{Q}^{2}$ & $122(3)$ \\
\hline O00L-W001-O2 ${ }^{1}$ & $161.2(13)$ & O1-P008-O11 & 180 & O14-W004-O00B ${ }^{1}$ & $101.5(11)$ & $\mathrm{C} 00 \mathrm{X}-\mathrm{C} 00 \mathrm{M}-\mathrm{C} 00 \mathrm{M}^{2}$ & $121(3)$ \\
\hline O00L-W001-O00A & $104.0(11)$ & O3 ${ }^{1}-\mathrm{P} 008-\mathrm{O} 1$ & 74(2) & O14-W004-O3 ${ }^{1}$ & $160.5(14)$ & C00X-C00M-C011 & $122(3)$ \\
\hline O00L-W001-O4 & $161.0(13)$ & O3-P008-O1 & $106(2)$ & O14-W004-O00K & $102.7(12)$ & $\mathrm{C} 011-\mathrm{C} 00 \mathrm{M}-\mathrm{C} 00 \mathrm{M}^{2}$ & $117(4)$ \\
\hline O00L-W001-O00B & $100.1(11)$ & O3-P008-O1 ${ }^{1}$ & $74(2)$ & O14-W004-O00O & $101.5(12)$ & C00I2-C00Q-Br07 & $113(2)$ \\
\hline O00L-W001-O00C & $102.1(11)$ & $\mathrm{O}^{1}{ }^{1-P 008-O} 1^{1}$ & $106(2)$ & O14-W004-O00S & $103.3(12)$ & C00I2-C00Q-C00X & 119(3) \\
\hline O00L-W001-O00D & 103.2(11) & $\mathrm{O}^{1}-\mathrm{P} 008-\mathrm{O} 3$ & $180(3)$ & $\mathrm{O} 00 \mathrm{H}-\mathrm{W} 005-\mathrm{O} 1$ & $158.8(13)$ & C00X-C00Q-Br07 & $128(2)$ \\
\hline O00A-W002-O21 & $63.0(11)$ & W001는 $-\mathrm{W} 002^{1}$ & $92.0(14)$ & O00H-W005-O00N1 & $102.9(12)$ & N012-C00V-C00Z & 106(3) \\
\hline O00A-W002-O31 & 93.1(11) & P008-O2-W001 ${ }^{1}$ & $129(2)$ & O00H-W005-O00S & $102.2(11)$ & N012-C00V-C01C & 124(3) \\
\hline O00A-W002-O00O & $156.0(10)$ & P008-O2-W002 ${ }^{1}$ & $125(2)$ & O00H-W005-O00Y1 & $102.3(12)$ & C00Z-C00V-C01C & $130(3)$ \\
\hline O00A-W002-O00U & $89.6(10)$ & $\mathrm{P} 008-\mathrm{O} 2-\mathrm{O} 4^{1}$ & $57(2)$ & O00H-W005-O010 & $101.8(11)$ & C00M-C00X-C00Q & $120(3)$ \\
\hline O00A-W002-O010 & $87.3(10)$ & $\mathrm{O} 4^{1}-\mathrm{O} 2-\mathrm{W} 001^{1}$ & $72(2)$ & O00N1-W005-O1 & $64.0(12)$ & C00M-C00X-C013 & 121(3) \\
\hline $\mathrm{O} 3^{1}-\mathrm{W} 002-\mathrm{O} 2^{1}$ & $40.2(12)$ & $\mathrm{O} 4^{1}-\mathrm{O} 2-\mathrm{W} 002^{1}$ & $136(3)$ & O00N1-W005-O00Y1 & $85.3(11)$ & C00Q-C00X-C013 & $119(3)$ \\
\hline $\mathrm{O} 00 \mathrm{O}-\mathrm{W} 002-\mathrm{O} 2^{1}$ & $93.9(12)$ & W002-O00A-W001 & $140.1(12)$ & O00S-W005-O1 & $91.5(12)$ & C00V-C00Z-N00F & $123(3)$ \\
\hline O00O-W002-O3 ${ }^{1}$ & $63.3(11)$ & W001-O4-W004 ${ }^{1}$ & $91.0(13)$ & O00S-W005-O00N1 & $154.9(11)$ & C018-C00Z-N00F & $129(3)$ \\
\hline O00P-W002-O21 & $159.6(12)$ & P008-O4-W001 & $128(2)$ & O00S-W005-O00Y1 & $88.0(11)$ & C018-C00Z-C00V & $109(3)$ \\
\hline O00P-W002-O00A & $101.9(11)$ & P008-O4-W004' & $125(2)$ & O00S-W005-O010 & 88.0(11) & C00I-C011-C00E & 121(3) \\
\hline O00P-W002-O3 ${ }^{1}$ & $160.2(12)$ & $\mathrm{P} 008-\mathrm{O} 4-\mathrm{O} 2^{1}$ & $57(2)$ & O00Y1-W005-O1 & $61.7(12)$ & C00I-C011-C00M & $121(3)$ \\
\hline O00P-W002-O00O & $102.0(11)$ & O2'-O4-W001 & 71(2) & O010-W005-O1 & $94.7(12)$ & C00M-C011-C00E & $118(3)$ \\
\hline O00P-W002-O00U & $100.4(12)$ & $\mathrm{O} 2^{1}-\mathrm{O} 4-\mathrm{W} 0041$ & $135(3)$ & O010-W005-O00N1 & $88.3(11)$ & O00G-C013-N00F & $119(3)$ \\
\hline O00P-W002-O010 & $101.7(12)$ & W001-O00B-W004' & $138.9(12)$ & O010-W005-O00Y1 & $155.9(11)$ & O00G-C013-C00X & $125(3)$ \\
\hline O00U-W002-O21 & $67.5(11)$ & W005-O1-W003 ${ }^{1}$ & 91.1(14) & O00C-W006-O11 & $91.9(12)$ & N00F-C013-C00X & 116(3) \\
\hline O00U-W002-O31 & $92.4(12)$ & W006'-O1-W003' & $92.8(13)$ & O00C-W006-O00U & $86.6(10)$ & N00W-C018-C01B & $120(3)$ \\
\hline O00U-W002-O00O & 87.1(10) & W006 ${ }^{1}-\mathrm{O} 1-\mathrm{W} 005$ & $93.0(13)$ & O00J-W006-O11 & $160.3(14)$ & C00Z-C018-N00W & 108(3) \\
\hline O00U-W002-O010 & $157.9(10)$ & P008-O1-W003 ${ }^{1}$ & $122(2)$ & O00J-W006-O00C & $102.9(11)$ & C00Z-C018-C01B & 132(3) \\
\hline O010-W002-O2 ${ }^{1}$ & $91.7(12)$ & P008-O1-W005 & $123(2)$ & O00J-W006-O00R & $105.7(12)$ & C30-N20-C34 & $114(5)$ \\
\hline O010-W002-O3 ${ }^{1}$ & $65.9(12)$ & P008-O1-W006 ${ }^{1}$ & $126(2)$ & O00J-W006-O00U & $100.2(12)$ & C30-N20-C38 & $131(6)$ \\
\hline O010-W002-O00O & $86.8(10)$ & P008-O1-O31 & $52.0(18)$ & O00J-W006-O00Y & $102.4(12)$ & C34-N20-C38 & $113(5)$ \\
\hline $\mathrm{O} 00 \mathrm{D}^{1}-\mathrm{W} 003-\mathrm{O} 1^{1}$ & $93.7(11)$ & $\mathrm{O}^{1}-\mathrm{O} 1-\mathrm{W} 003^{1}$ & $130(2)$ & O00R-W006-O11 & $61.0(12)$ & C34-C01D-C01H & 101(5) \\
\hline O00D ${ }^{1}-W 003-O 00 K$ & $87.5(10)$ & O3'-O1-W005 & $70.7(18)$ & O00R-W006-O00C & $151.2(11)$ & C30-C01H-C01D & $102(4)$ \\
\hline O00K-W003-O1 ${ }^{1}$ & $91.0(11)$ & $\mathrm{O}^{1}{ }^{1}-\mathrm{O} 1-\mathrm{W} 006^{1}$ & $133(2)$ & O00R-W006-O00U & $85.7(10)$ & $\mathrm{O} 016-\mathrm{C} 30-\mathrm{C} 01 \mathrm{H}$ & $123(5)$ \\
\hline O00N-W003-O1 ${ }^{1}$ & $63.8(12)$ & W001-O00C-W006 & $142.2(13)$ & O00U-W006-O11 & $93.5(12)$ & N20-C30-O016 & $122(6)$ \\
\hline O00N-W003-O00D ${ }^{1}$ & $87.5(11)$ & W004'-O3-W002 & $92.5(13)$ & O00Y-W006-O11 & 64.1(13) & $\mathrm{N} 20-\mathrm{C} 30-\mathrm{C} 01 \mathrm{H}$ & $115(7)$ \\
\hline O00N-W003-O00K & $154.0(11)$ & P008-O3-W002 & $125(2)$ & O00Y-W006-O00C & $90.5(11)$ & N20-C34-C01D & 104(5) \\
\hline O00R-W003-O11 & $59.6(12)$ & P008-O3-W004 ${ }^{1}$ & $128(2)$ & O00Y-W006-O00R & 86.1(11) & C019-N017-C01A & $121(6)$ \\
\hline O00R-W003-O00D ${ }^{1}$ & $151.9(11)$ & $\mathrm{P} 008-\mathrm{O} 3-\mathrm{O} 1^{1}$ & $54.4(18)$ & O00Y-W006-O00U & $157.3(11)$ & C019-N017-C35 & $111(6)$ \\
\hline O00R-W003-O00K & $84.8(11)$ & $\mathrm{O} 1^{1}-\mathrm{O} 3-\mathrm{W} 002^{1}$ & $130(2)$ & O21-P008-O2 & $180(3)$ & C01A-N017-C35 & $127(5)$ \\
\hline O00R-W003-O00N & $87.7(11)$ & $\mathrm{O} 1^{1}-\mathrm{O} 3-\mathrm{W} 004^{1}$ & $129(2)$ & O2-P008-O1 & $72(2)$ & C39-C014-C35 & $106(5)$ \\
\hline O13-W003-O $1^{1}$ & $158.2(13)$ & W001-O00D-W0031 & $140.8(13)$ & O2-P008-O11 & $108(2)$ & O01K-C019-N017 & $126(7)$ \\
\hline
\end{tabular}




\begin{tabular}{ll|lr|lll|l} 
O13-W003-O00D & $103.4(11)$ & W004-O00K-W003 & $141.0(14)$ & O21-P008-O11 & 72(2) & O01K-C019-C39 & 125(7) \\
O13-W003-O00K & $103.0(11)$ & W005-O00N-W003 & $140.5(15)$ & O21-P008-O1 & $108(2)$ & N017-C019-C39 \\
O13-W003-O00N & $103.0(12)$ & W004-O00O-W002 & $138.9(14)$ & O2-P008-O3 & $70(2)$ & N017-C35-C014 \\
O13-W003-O00R & $104.6(12)$ & W003-O00R-W006 & $145.0(15)$ & O21-P008-O3 & $110(2)$ & C014-C39-C019 \\
O00B ${ }^{1-W 004-O 4 ~}$ & $64.0(11)$ & W005-O00S-W004 & $140.5(13)$ & & $105(6)$ & \\
\hline
\end{tabular}

12-X, 1-Y, 1-Z; ${ }^{2} 1-\mathrm{X}, 1-\mathrm{Y}, 1-\mathrm{Z}$

Table S5. Bond angles for the crystal 2

\begin{tabular}{|c|c|c|c|c|c|c|c|}
\hline Atom & Angle $/^{\circ}$ & Atom & Angle $/^{\circ}$ & Atom & Angle $/^{\circ}$ & Atom & Angle $/^{\circ}$ \\
\hline O00D-W001-O4 ${ }^{1}$ & $63.3(5)$ & O4-P008-O41 & $180.0(11)$ & $\mathrm{O}^{2} \mathrm{~S}^{1}-\mathrm{W} 003-\mathrm{O} 00 \mathrm{~W}$ & $87.3(6)$ & P008-O2-W0041 & $122.9(9)$ \\
\hline O00D-W001-O1 ${ }^{1}$ & $92.3(6)$ & O4-P008-O1 ${ }^{1}$ & $115.0(9)$ & O00S ${ }^{1}-\mathrm{W} 003-O 1^{1}$ & $94.7(7)$ & P008-O2-W005 & $119.6(9)$ \\
\hline O00E-W001-O00D & $87.7(5)$ & O4-P008-O1 & $65.0(9)$ & $\mathrm{O}^{0} \mathrm{~S}^{1}-\mathrm{W} 003-\mathrm{O} 3$ & $62.1(7)$ & P008-O2-W006 ${ }^{1}$ & $124.7(8)$ \\
\hline O00E-W001-O00L & $87.4(6)$ & $\mathrm{O}^{1}-\mathrm{P} 008-\mathrm{O} 1$ & $115.0(9)$ & O00W-W003-O $1^{1}$ & $89.8(7)$ & $\mathrm{P} 008-\mathrm{O} 2-\mathrm{O} 4^{1}$ & $51.4(8)$ \\
\hline O00E-W001-O4 ${ }^{1}$ & $90.4(6)$ & $\mathrm{O} 4^{1}-\mathrm{P} 008-\mathrm{O} 1^{1}$ & $65.0(9)$ & O00W-W003-O3 & $66.0(6)$ & $\mathrm{O}^{1}{ }^{1}-\mathrm{O} 2-\mathrm{W} 004^{1}$ & $135.2(10)$ \\
\hline O00E-W001-O1 ${ }^{1}$ & $65.6(6)$ & $\mathrm{O} 4-\mathrm{P} 008-\mathrm{O} 3^{1}$ & $113.2(8)$ & O3-W003-O $1^{1}$ & $40.9(6)$ & $\mathrm{O} 4^{1}-\mathrm{O} 2-\mathrm{W} 005^{1}$ & $130.2(10)$ \\
\hline O00F-W001-O00D & $85.8(5)$ & $\mathrm{O} 4{ }^{1}-\mathrm{P} 008-\mathrm{O} 3$ & $113.2(8)$ & O00E-W004-O00O & $87.2(5)$ & $\mathrm{O} 4^{1}-\mathrm{O} 2-\mathrm{W} 006^{1}$ & $73.3(8)$ \\
\hline O00F-W001-O00E & $154.0(6)$ & $\mathrm{O} 4^{1}-\mathrm{P} 008-\mathrm{O} 3^{1}$ & $66.8(8)$ & O00E-W004-O2 ${ }^{1}$ & $94.5(6)$ & $\mathrm{N} 01^{1}-\mathrm{N} 010-\mathrm{C} 017$ & $116.8(16)$ \\
\hline O00F-W001-O00L & $88.3(6)$ & O4-P008-O3 & $66.8(8)$ & O00E-W004-O016 & $153.9(7)$ & N010-N011-C018 & $101.8(18)$ \\
\hline O00F-W001-O4 ${ }^{1}$ & $64.2(6)$ & $\mathrm{O} 1-\mathrm{P} 008-\mathrm{O} 2^{1}$ & $105.6(9)$ & O00H-W004-O00E & $104.1(6)$ & W004-O012-W005 & $140.8(8)$ \\
\hline O00F-W001-O ${ }^{1}$ & $89.5(6)$ & $\mathrm{O} 1^{1}-\mathrm{P} 008-\mathrm{O} 2^{1}$ & $74.4(9)$ & O00H-W004-O00O & $102.8(6)$ & W005-O014-W006 & $141.3(9)$ \\
\hline O00L-W001-O00D & $156.1(6)$ & $\mathrm{O} 1^{1}-\mathrm{P} 008-\mathrm{O} 2$ & $105.6(9)$ & O00H-W004-O $2^{1}$ & $157.1(7)$ & C017-C015-N00G & $124.9(18)$ \\
\hline O00L-W001-O4 ${ }^{1}$ & $93.3(6)$ & O1-P008-O2 & $74.4(9)$ & O00H-W004-O012 & $102.5(7)$ & C017-C015-C018 & $108.4(17)$ \\
\hline O00L-W001-O1 ${ }^{1}$ & $64.4(6)$ & O1-P008-O1 ${ }^{1}$ & 180 & O00H-W004-O016 & $102.0(7)$ & C018-C015-N00G & $126.7(15)$ \\
\hline O00X-W001-O00D & $102.4(6)$ & O1-P008-O3 & $110.8(9)$ & O00O-W004-O $2^{1}$ & $91.3(6)$ & W006-O016-W004 & $141.6(10)$ \\
\hline O00X-W001-O00E & $102.9(7)$ & O1-P008-O3 ${ }^{1}$ & $69.2(9)$ & O012-W004-O00E & $89.5(6)$ & N010-C017-C20 & $125.4(17)$ \\
\hline O00X-W001-O00F & $103.0(7)$ & $\mathrm{O} 1^{1}-\mathrm{P} 008-\mathrm{O} 3$ & $69.2(9)$ & O012-W004-O00O & $154.5(6)$ & C015-C017-N010 & $101.3(18)$ \\
\hline O00X-W001-O00L & $101.5(7)$ & $\mathrm{O} 1^{1}-\mathrm{P} 008-\mathrm{O} 3^{1}$ & $110.8(9)$ & O012-W004-O21 & $63.8(6)$ & C015-C017-C20 & $133(2)$ \\
\hline O00X-W001-O4 & $160.3(7)$ & O3-P008-O2 & $77.3(10)$ & O012-W004-O016 & $86.8(6)$ & N011-C018-C015 & $111.6(18)$ \\
\hline O00X-W001-O $1^{1}$ & $161.2(7)$ & $\mathrm{O}^{1}{ }^{1}-\mathrm{P} 008-\mathrm{O} 2$ & $102.7(10)$ & O016-W004-O00O & $85.1(7)$ & N011-C018-C0AA & $117(2)$ \\
\hline $\mathrm{O} 1^{1}-\mathrm{W} 001-\mathrm{O} 4^{1}$ & $38.5(6)$ & $\mathrm{O} 3-\mathrm{P} 008-\mathrm{O} 2^{1}$ & $102.7(10)$ & O016-W004-O21 & $60.8(7)$ & C015-C018-C0AA & $131.4(16)$ \\
\hline O00D-W002-O00M & $89.9(6)$ & $\mathrm{O} 3^{1}-\mathrm{P} 008-\mathrm{O} 2^{1}$ & $77.3(10)$ & O00C-W005-O00M & $101.3(6)$ & W001'-O4-W002 ${ }^{1}$ & $91.9(5)$ \\
\hline O00D-W002-O00S & $155.5(7)$ & O3-P008-O3 ${ }^{1}$ & $180.0(12)$ & O00C-W005-O00W ${ }^{1}$ & $100.8(6)$ & P008-O4-W001 ${ }^{1}$ & $126.4(9)$ \\
\hline O00D-W002-O4 ${ }^{1}$ & $63.4(5)$ & W002-O00D-W001 & $140.9(7)$ & $\mathrm{O} 00 \mathrm{C}-\mathrm{W} 005-\mathrm{O} 2^{1}$ & $158.0(7)$ & P008-O4-W002 ${ }^{1}$ & $126.3(9)$ \\
\hline O00D-W002-O3 ${ }^{1}$ & $94.6(6)$ & W001-O00E-W004 & $141.4(7)$ & O00C-W005-O012 & $103.3(6)$ & $\mathrm{P} 008-\mathrm{O} 4-\mathrm{O} 21$ & $57.1(8)$ \\
\hline $\mathrm{O}^{0} \mathrm{I}^{1}-\mathrm{W} 002-\mathrm{O} 00 \mathrm{D}$ & $85.7(6)$ & W006 ${ }^{1}-\mathrm{O} 00 \mathrm{~F}-\mathrm{W} 001$ & $142.8(7)$ & O00C-W005-O014 & $103.1(7)$ & P008-O4-O1 & $57.9(9)$ \\
\hline $\mathrm{O} 00 \mathrm{I}^{1}-\mathrm{W} 002-\mathrm{O} 00 \mathrm{M}$ & $154.7(7)$ & C00T-N00G-C00V & $124.1(11)$ & O00M-W005-O00W ${ }^{1}$ & $88.7(6)$ & P008-O4-O3 & $58.0(8)$ \\
\hline O00I ${ }^{1}-\mathrm{W} 002-\mathrm{O} 00 \mathrm{~S}$ & $86.8(6)$ & C00T-N00G-C015 & $120.4(12)$ & $\mathrm{O} 00 \mathrm{M}-\mathrm{W} 005-\mathrm{O} 2^{1}$ & $94.5(7)$ & $\mathrm{O} 2^{1}-\mathrm{O} 4-\mathrm{W} 001^{1}$ & $129.3(9)$ \\
\hline O001 ${ }^{1}-\mathrm{W} 002-\mathrm{O} 4^{1}$ & $64.8(6)$ & C015-N00G-C00V & $115.4(12)$ & O00M-W005-O012 & $86.5(6)$ & $\mathrm{O} 2^{1}-\mathrm{O} 4-\mathrm{W} 002^{1}$ & $128.3(10)$ \\
\hline $\mathrm{O}^{0} \mathrm{I}^{1}-\mathrm{W} 002-\mathrm{O} 3^{1}$ & $89.6(7)$ & W002-O00I-W006 & $142.0(8)$ & O00M-W005-O014 & $155.5(7)$ & O1-O4-W001 ${ }^{1}$ & $68.5(8)$ \\
\hline O00M-W002-O4 ${ }^{1}$ & $91.0(7)$ & C00R2-C00J-C00K & $120.2(13)$ & $\mathrm{O}_{00} \mathrm{~W}^{1}-\mathrm{W} 005-\mathrm{O} 2^{1}$ & $94.6(6)$ & $\mathrm{O} 1-\mathrm{O} 4-\mathrm{W} 002^{1}$ & $135.6(11)$ \\
\hline
\end{tabular}




\begin{tabular}{|c|c|c|c|c|c|c|c|}
\hline O00M-W002-O3 ${ }^{1}$ & $65.8(6)$ & $\mathrm{C} 00 \mathrm{~J}-\mathrm{C} 00 \mathrm{~K}-\mathrm{C} 00 \mathrm{Q}$ & $121.8(13)$ & $\mathrm{O}^{0} \mathrm{~W}^{1}-\mathrm{W} 005-\mathrm{O} 012$ & $155.8(7)$ & $\mathrm{O} 1-\mathrm{O} 4-\mathrm{O} 2^{1}$ & $92.6(11)$ \\
\hline O00S-W002-O00M & $87.0(6)$ & $\mathrm{C} 00 \mathrm{~J}-\mathrm{C} 00 \mathrm{~K}-\mathrm{C} 00 \mathrm{~T}$ & $118.5(12)$ & $\mathrm{O}^{2} 0 \mathrm{~W}^{1}-\mathrm{W} 005-\mathrm{O} 014$ & $88.8(7)$ & O1-O4-O3 & $99.7(14)$ \\
\hline O00S-W002-O4 ${ }^{1}$ & $92.4(6)$ & $\mathrm{C} 00 \mathrm{Q}-\mathrm{C} 00 \mathrm{~K}-\mathrm{C} 00 \mathrm{~T}$ & $119.7(12)$ & O012-W005-O2 ${ }^{1}$ & $62.3(6)$ & O3-O4-W001 ${ }^{1}$ & $138.3(11)$ \\
\hline O00S-W002-O3 ${ }^{1}$ & $62.0(7)$ & W001-O00L-W003 & $139.5(9)$ & O014-W005-O2 ${ }^{1}$ & $61.4(7)$ & O3-O4-W002 ${ }^{1}$ & $68.3(8)$ \\
\hline O15-W002-O00D & $101.4(6)$ & W005-O00M-W002 & $140.2(8)$ & O014-W005-O012 & $85.9(6)$ & $\mathrm{O} 3-\mathrm{O} 4-\mathrm{O} 2^{1}$ & $89.7(11)$ \\
\hline O15-W002-O00I ${ }^{1}$ & $101.0(7)$ & W003-O00O-W004 & $140.8(7)$ & $\mathrm{O}^{0} \mathrm{~F}^{1}-\mathrm{W} 006-\mathrm{O} 00 \mathrm{I}$ & $85.8(5)$ & W0011-O1-W003 ${ }^{1}$ & $93.1(6)$ \\
\hline O15-W002-O00M & $104.3(7)$ & $\mathrm{C} 00 \mathrm{Q}-\mathrm{C} 00 \mathrm{P}-\mathrm{C} 00 \mathrm{~V}$ & $118.2(12)$ & $\mathrm{O} 00 \mathrm{~F}^{1}-\mathrm{W} 006-\mathrm{O} 2^{1}$ & $92.4(6)$ & P008-O1-W001 & $130.1(10)$ \\
\hline O15-W002-O00S & $103.0(6)$ & C00R-C00P-C00Q & $119.3(13)$ & $\mathrm{O}^{0} \mathrm{~F}^{1}-\mathrm{W} 006-\mathrm{O} 014$ & $86.9(6)$ & P008-O1-W003 & $123.6(9)$ \\
\hline O15-W002-O41 & $158.7(6)$ & $\mathrm{COOR}-\mathrm{C} 00 \mathrm{P}-\mathrm{C} 00 \mathrm{~V}$ & $122.5(12)$ & O00I-W006-O2 ${ }^{1}$ & $92.2(6)$ & P008-O1-O4 & $57.1(8)$ \\
\hline O15-W002-O31 & $161.4(6)$ & C00K-C00Q-C00P & $123.2(13)$ & O00U-W006-O00F1 & $103.5(7)$ & P008-O1-O31 & $56.4(8)$ \\
\hline O31-W002-O4 ${ }^{1}$ & $39.7(5)$ & $\mathrm{C} 00 \mathrm{~K}-\mathrm{C} 00 \mathrm{Q}-\mathrm{C} 00 \mathrm{Q}^{2}$ & $118.3(14)$ & O00U-W006-O00I & $103.1(7)$ & O4-O1-W001 ${ }^{1}$ & 73.1(9) \\
\hline O00L-W003-O00W & $85.9(6)$ & C00P-C00Q-C00Q ${ }^{2}$ & $118.5(15)$ & O00U-W006-O2 ${ }^{1}$ & $158.5(7)$ & O4-O1-W003 ${ }^{1}$ & $139.3(12)$ \\
\hline O00L-W003-O1 ${ }^{1}$ & $61.8(6)$ & 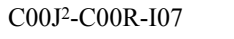 & $112.7(10)$ & O00U-W006-O014 & $102.8(8)$ & $\mathrm{O} 4-\mathrm{O} 1-\mathrm{O} 3^{1}$ & $98.4(13)$ \\
\hline O00L-W003-O3 & $93.8(6)$ & $\mathrm{C}^{2} 00 \mathrm{~J}^{2}-\mathrm{C} 00 \mathrm{R}-\mathrm{C} 00 \mathrm{P}$ & $121.8(12)$ & O00U-W006-O016 & $103.1(8)$ & $\mathrm{O}^{1}-\mathrm{O} 1-\mathrm{W} 001^{1}$ & $139.5(12)$ \\
\hline O00N-W003-O00L & $101.5(6)$ & C00P-C00R-I07 & $125.5(11)$ & O014-W006-O00I & $154.0(7)$ & $\mathrm{O}^{1}-\mathrm{O} 1-\mathrm{W} 003^{1}$ & $67.3(8)$ \\
\hline O00N-W003-O00O & $102.7(7)$ & W0031-O00S-W002 & $140.3(10)$ & O014-W006-O21 & $63.2(6)$ & W002 1 -O3-W003 & $94.6(7)$ \\
\hline O00N-W003-O00S ${ }^{1}$ & $102.9(7)$ & O00B-C00T-N00G & $117.9(13)$ & O016-W006-O00F ${ }^{1}$ & $153.4(7)$ & P008-O3-W002 ${ }^{1}$ & $127.0(9)$ \\
\hline O00N-W003-O00W & $102.7(7)$ & O00B-C00T-C00K & $124.1(12)$ & O016-W006-O00I & $88.9(6)$ & P008-O3-W003 & $126.3(9)$ \\
\hline O00N-W003-O1 ${ }^{1}$ & $158.7(6)$ & N00G-C00T-C00K & $118.0(12)$ & O016-W006-O2 ${ }^{1}$ & $61.8(7)$ & P008-O3-O4 & $55.2(8)$ \\
\hline O00N-W003-O3 & $160.4(6)$ & O00A-C00V-N00G & $118.0(13)$ & O016-W006-O014 & $86.6(6)$ & P008-O3-O1 ${ }^{1}$ & $54.5(8)$ \\
\hline O00O-W003-O00L & $88.2(6)$ & $\mathrm{O} 00 \mathrm{~A}-\mathrm{C} 00 \mathrm{~V}-\mathrm{C} 00 \mathrm{P}$ & $125.3(14)$ & $\mathrm{O} 2-\mathrm{P} 008-\mathrm{O} 2^{1}$ & $180.0(8)$ & $\mathrm{O} 4-\mathrm{O} 3-\mathrm{W} 002^{1}$ & $71.9(8)$ \\
\hline O00O-W003-O00S ${ }^{1}$ & $87.9(6)$ & N00G-C00V-C00P & $116.7(11)$ & $\mathrm{O} 4-\mathrm{P} 008-\mathrm{O} 2$ & $108.5(9)$ & O4-O3-W003 & $139.7(13)$ \\
\hline O00O-W003-O00W & $154.6(6)$ & W005'-O00W-W003 & $139.7(7)$ & $\mathrm{O}^{1}{ }^{-}-\mathrm{P} 008-\mathrm{O} 2$ & $71.5(9)$ & $\mathrm{O} 4-\mathrm{O} 3-\mathrm{O} 1^{1}$ & $96.0(12)$ \\
\hline O00O-W003-O $1^{1}$ & $65.8(6)$ & W004'-O2-W0051 & $92.9(6)$ & $\mathrm{O} 4^{1}-\mathrm{P} 008-\mathrm{O} 2^{1}$ & $108.5(9)$ & $\mathrm{O} 1^{1}-\mathrm{O} 3-\mathrm{W} 002^{1}$ & $143.1(13)$ \\
\hline O00O-W003-O3 & $89.8(6)$ & W006 ${ }^{1}-\mathrm{O} 2-\mathrm{W} 004^{1}$ & $95.1(7)$ & $\mathrm{O} 4-\mathrm{P} 008-\mathrm{O} 2^{1}$ & $71.5(9)$ & $\mathrm{O} 1^{1}-\mathrm{O} 3-\mathrm{W} 003$ & $71.9(8)$ \\
\hline $\mathrm{O}^{1} \mathrm{~S}^{1}-\mathrm{W} 003-\mathrm{O} 00 \mathrm{~L}$ & $155.5(7)$ & W006 1 -O2-W005 & $93.8(7)$ & & & & \\
\hline
\end{tabular}

${ }^{1} 1-X, 1-Y, 1-Z ;{ }^{2} 2-X, 1-Y, 1-Z$

\section{References}

(1) Sarkar, A.; Dhiman, S.; Chalishazar, A.; George, S. J., Visualization of Stereoselective Supramolecular Polymers by Chirality-Controlled Energy Transfer. Angew. Chem. Int. Ed. 2017, 56, $13767-13771$

(2) Wade, C. R.; Li, M.; Dinca, M., Facile deposition of multicolored electrochromic metal-organic framework thin films. Angew. Chem. Int. Ed. 2013, 52, 13377-81. 
(3) Dawson, R. E.; Hennig, A.; Weimann, D. P.; Emery, D.; Ravikumar, V.; Montenegro, J.; Takeuchi, T.; Gabutti, S.; Mayor, M.; Mareda, J.; Schalley, C. A.; Matile, S., Experimental evidence for the functional relevance of anion-pi interactions. Nat. Chem. 2010, 2, 533-538.

(4) Farrugia, L. J., WinGXandORTEP for Windows: an update. J. Appl. Cryst. 2012, 45, 849-854.

(5) Sheldrick, G. M., SHELXT - integrated space-group and crystal-structure determination. Acta Crystallogr A Found Adv. 2015, 71, 3-8.

(6) Sheldrick, G. M., Crystal structure refinement with SHELXL. Acta Crystallogr C Struct Chem. 2015, $71,3-8$.

(7) Frisch, M. J.; Trucks, G. W.; Schlegel, H. B.; Scuseria, G. E.; Robb, M. A.; Cheeseman, J. R.; Scalmani, G.; Barone, V.; Mennucci, B.; Petersson, G. A.; Nakatsuji, H.; Caricato, M.; Li, X.; Hratchian, H. P.; Izmaylov, A. F.; Bloino, J.; Zheng, G.; Sonnenberg, J. L.; Hada, M.; Ehara, M.; Toyota, K.; Fukuda, R.; Hasegawa, J.; Ishida, M.; Nakajima, T.; Honda, Y.; Kitao, O.; Nakai, H.; Vreven, T.; Montgomery, Jr., J. A.; Peralta, J. E.; Ogliaro, F.; Bearpark, M.; Heyd, J. J.; Brothers, E.; Kudin, K. N.; Staroverov, V. N.; Kobayashi, R.; Normand, J.; Raghavachari, K.; Rendell, A.; Burant, J. C.; Iyengar, S. S.; Tomasi, J.; Cossi, M.; Rega, Millam, N. J.; Klene, M.; Knox, J. E.; Cross, J. B.; Bakken, V.; Adamo, C.; Jaramillo, J.; Gomperts, R. E.; Stratmann, O.; Yazyev, A. J.; Austin, R.; Cammi, C.; Pomelli, J. W.; Ochterski, R.; Martin, R. L.; Morokuma, K.; Zakrzewski, V. G.; Voth, G. A.; Salvador, P.; Dannenberg, J. J.; Dapprich, S.; Daniels, A. D.; Farkas, O.; Foresman, J. B.; Ortiz, J. V.; Cioslowski, J.; Fox, D. J. Gaussian 09, Revision A.01; Gaussian Inc., Wallingford CT, 2009.

(8) Hao, P.; Zhu, H.; Pang, Y.; Shen, J.; Fu, Y., The impact of positional isomerism on electronic and photochromic properties of 1D zinc-based naphthalene diimide coordination polymers. CrystEngComm 2020, 22, 3371-3377. 